\title{
Protée
}

\section{Kasimir Malevitch, Maurice Blanchot. Le silence de l'oeuvre}

\section{Emmanuelle Ravel}

Volume 28, numéro 2, 2000

\section{Le silence}

URI : https://id.erudit.org/iderudit/030595ar

DOI : https://doi.org/10.7202/030595ar

Aller au sommaire du numéro

\section{Éditeur(s)}

Département des arts et lettres - Université du Québec à Chicoutimi

\section{ISSN}

0300-3523 (imprimé)

1708-2307 (numérique)

Découvrir la revue

Citer cet article

Ravel, E. (2000). Kasimir Malevitch, Maurice Blanchot. Le silence de l'oeuvre. Protée, 28(2), 69-78. https://doi.org/10.7202/030595ar

\section{Résumé de l'article}

C'est sans doute le terme de "suprématiste " qui conviendrait le mieux pour décrire le travail de Maurice Blanchot à cause de ce qui se donne comme un thème récurrent dans toutes ses publications : le défi qu'il s'est lancé de considérer comme absolues les limites de la représentation. Dans l'un ou l'autre de ses ouvrages, l'abstraction du langage devient paradoxalement le genre suprême de la qualité concrète, en peinture aussi bien qu'en littérature, de sa praxis. À ce stade, les enjeux naissent de la crise qui affecte une avant-garde qui ne parvient plus à soutenir les signes de renouvellement perpétuel de la forme de sa création. Entre les années 1910 et 1913, l'aporie de la modernité conduit rapidement à la formulation de l'idée de la mort de l'art et, après la deuxième guerre mondiale, avec l'apparition de $L$ 'Espace littéraire de Blanchot, cette même aporie amène à engager la littérature dans l'expérience des limites. Cet article se propose donc d'évaluer, par le biais de la présence du silence dans l'oeuvre, le degré d'aridité qui affecte la représentation, de celle des sens en particulier, et les limites que peuvent atteindre de telles aventures. 


\section{KASIMIR M ALEVITCH, MAURICE BLANCHOT LE SILEN CE DE L'GUVRE}
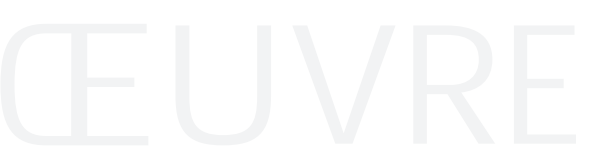

EM M AN U ELLE RAVEL

L'intensité est silencieuse. Son image ne l'est pas.

(J'aime qui m'éblouit puis accentue l'obscur à l'intérieur de moi.)

René Char, Rougeur des Matinaux.

Peut-on penser l'abstraction en peinture - celle de l'image - et regarder celle de l'écriture - qui tisse le texte blanchotien? Le lieu de l'abstraction est-il celui des sens, du sens, ou encore du non-sens? L'espace pictural se veut-il être lu, le langage de l'œuvre de Blanchot est-il immédiatement image? C'est à travers ce florilège de questions que nous sommes appelés à nous mouvoir, convoqués par deux présences intriquées mais irréductibles l'une à l'autre, celle de la peinture blanc sur blanc, celle de l'écriture blanche sur la page blanche. Comme nous essayons de le suggérer, la disparition sera au centre de notre réflexion, inachevant indéfiniment la répudiation du signe. L'absence est-elle parlable, y a-t-il ou non fixité de l'image comme du langage, c'est-à-dire silence en peinture, silence en littérature? Notre but sera d'évaluer le degré de tarissement de l'une et l'autre œuvre, celle de Blanchot par une toile de Malevitch et réciproquement, pour dire enfin si silence, non-concept et abstraction s'équivalent dans le spéculum image/ langage.

L'abstraction en peinture voit le jour grâce aux premières aquarelles de Kandinsky, en 1910. Période florissante de l'Avant-Garde, trois ans après les chères Demoiselles d'Avignon, l'idée de l'abstraction (déjà notre questionnement se profile dans la coexistence de ces deux termes) naît du rejet de la représentation, d'un dégoût de l'illusion. Les années qui suivront, nous parlons de 1912-1913, seront le théâtre de l'explosion de cette révolution, dans tous les arts, de cette dislocation, cacophonie entre forme et contenu. La Recherche du temps perdu, le Pierrot lunaire de Schönberg, le premier Ready-made de Duchamp, les Calligrammes d'Apollinaire... autant de remises en question du statut de la subjectivité (dépossession de l'artiste et perte de sa souveraineté; destruction de la domination organique de l'œuvre; présentation de son hétérogénéité, de sa pluralité; désir de saturer l'espace baigné 
d'aura, de briser la forme...). L'œuvre s'accole au trauma, au fragmentaire, à la folie qui est chez Michel Foucault, dans son Histoire de la folie à l'âge classique, liée à l'absence d'œuvre. Le désœuvrement étant, il faut le répéter sans cesse, l'absolu unique, saillant et déchirant, où s'abîme la modernité.

De Schönberg à Kandinsky ou Malevitch, c'est cette critique du Cogito qui justifie l'avant-garde, s'il est vrai, comme y insiste Malevitch, qu'on ne pourra créer des formes esthétiques radicalement nouvelles qu'après avoir «supprimé de tous nos arts l'idée petite-bourgeoise du sujet» et «tapé sur la conscience comme sur des clous que l'on enfonce dans un mur de pierre». 1

Essayons à présent de découvrir le rapport étroit qui existe entre l'abstraction, le refus de la figuration et la position de l'image, telle qu'elle se donne à voir dans le Carré blanc sur fond blanc, l'œuvre célèbre de 1918. Ceci pourra nous conduire à la question du langage en procès de la représentation du monde. Afin que l'œuvre d'art moderniste acquière une existence autonome, il a fallu qu'elle se débarrasse de son aliénation subjectiviste. L'art abstrait à ses débuts s'est surtout prévalu de l'expression du monde intérieur, de la sensibilité de l'artiste: Kandinsky, Klee, Mondrian ont préludé à son expansion par l'éloquence de lignes, de points et de couleurs. Les préoccupations de Malevitch se réclamaient plutôt d'une abstraction suprême, ayant pour nom Suprématisme, dont la technique dépassait largement l'utilisation et l'exposition d'un langage personnel. L'intention du peintre était d'exposer ses tableaux sans cadres, suggérant ainsi que leur espace déborde à l'infini le champ de la perception. Issu d'une théorie esthétique qui, pensée à l'apogée de la révolution bolchévique, se devait d'être avant-gardiste, le Suprématisme se voulut une sorte d'apothéose du futurisme, épilogue de la plasticité parfaite. Notre attention se penchera sur l'avènement de la pensée du Monde sans objet, théorie que Malevitch manifestera publiquement en décembre 1915 à l'occasion de l'exposition « 0,10 », organisée à Petrograd, deux ans après l'élaboration de sa toile Carré noir. Hanté par la philosophie de Schopenhauer, l'artiste russe peint pour retrouver sur sa toile le frémissement d'un monde dénué de cette "volonté de représentation», pour rendre justice à l'espace plutôt qu'à ce qui l'encombre en lui rendant un possible lieu d'existence: la surface plane. «Pour l'instant, la voie de l'homme passe par l'espace, le Suprématisme est le sémaphore de la couleur dans son abîme infini", proclame-t-il (Écrits, p.83). Le projet de l'émancipation du fond et de la forme est à cette époque le souci majeur de tous les artistes d'avant-garde, pour qui la couleur doit à tout prix s'affranchir du tracé. C'est dans cette dissociation - mise à mort de la chose par le mot, selon l'héritage de Mallarmé, telle qu'elle se trouve à l'œuvre également à l'intérieur du texte littéraire chez Blanchot-que nous examinerons la présence du silence, degré zéro (ou ultime?) de la création. Le passage à l'abstraction pour Malevitch s'est donc opéré par le biais de la relation objectale. Celle-ci a eu lieu dans l'espace de la négativité puisqu'il a souhaité mettre en valeur «le sentiment de l'absence d'objet». L'enjeu ici est encore de montrer la perte, la disparition, et nous serons amené à nous interroger sur l'effective disparition de ce qui enfreint l'abstraction suprême. Ce travail sur l'image s'affilie étroitement à l'approche blanchotienne du langage dans l'espace littéraire, à son repérage par rapport au noyau central de l'œuvre qui est le fameux point dissident de toute représentation.

Le Suprématisme avait besoin de franchir le lieu de la dialectique qui enfermait l'image dans la tension, illustrée par le mythe d'Orphée dans L'Espace littéraire de Maurice Blanchot, de l'apparence et de l'apparition. L'a-t-il dépassé ou s'est-il maintenu sous sa dictée? Ceci restera une de nos prérogatives majeures que de dire si oui ou non, le silence a éclos, la mort est advenue. Évoquer un possible éloignement de cette dialectique, c'est effectivement poser la négativité comme affirmation, porter l'absence au-delà de la lutte des formes et des couleurs, des débats sur la représentation. C'est postuler un lieu autre, toujours en dehors. L'image est la mort, certes; de la figuration, elle est la chose, l'Eurydice perdue, le cadavre. C'est qu'elle est langage; cela reste à éclaircir, nous ne 
l'oublions pas. Dire si ce langage est muet revient à poser cette question: comment le peintre avantgardiste russe parvient-il à rejoindre cette expérience blanchotienne du langage comme pure forme de l'absence? Mais Blanchot y parvient-il vraiment? Peuton dire du Carré blanc de Malevitch, comme du livre blanchotien, qu'il n'est pas écrit mais écriture, que ce tableau n'est point peint, mais peinture? Il faut en arriver à ce que Fernand Léger nommera un espace autofiguratif. Le défi que Malevitch s'impose de relever est anamorphique - il s'accompagne d'une transformation de la forme, si l'on peut oser le pléonasme - par rapport à celui de Blanchot qui est de faire taire le langage de l'œuvre en le rendant invisible. Le regard a d'emblée chez Blanchot un statut ordonnancier et légiférant: l'œuvre, qui est sa propre image (d'écriture, de peinture), doit en plus prendre la forme de la perte, comme si celle-ci se trouvait figurable par les mots ou le tracé de la toile.

Tout se joue donc dans la décision du regard. C'est dans cette décision que l'origine est approchée par la force du regard qui délie l'essence de la nuit, lève le souci, interrompt l'incessant en le découvrant: moment $d u$ désir, de l'insouciance et de l'autorité. ${ }^{2}$

L'œil se pose sur la scintillation - le permanent, l'apparence donc - et s'aveugle de ce qu'il advient de visible - l'apparition - et qui se perd, disparait. L'actuel donc. C'est en cette éternelle illumination, dans le détour et le retour où elle se confine, qu'il faut voir où se trouve l'absence. Absence et disparition de l'origine, celle-ci se signalant parce que l'image a perdu son original, l'autre chose. Il faut comprendre que dans cette altérité se cache le principe organisateur de l'œuvre. Comme l'explique J. Pfeiffer dans la revue Critique ${ }^{3}$ : Justement, ce que Blanchot dit de l'œuure, nous pouvons déjà le dire de l'image. Elle non plus n'existe pas comme un quelconque objet du monde, elle non plus ne saurait se laisser réduire à une quelconque réalité. Elle n'existe qu'en se dépassant vers autre chose, qu'en signifiant, par la vertu de la ressemblance, une autre chose qu'elle-même, qui est absente.

L'absence est certainement et paradoxalement le référent du texte. Elle va devenir référence, comprenons ici un passage à l'image. Là où nous l'avons suggéré, le simulacre fait office de vérité. Signifiant de l'écart originel, le silence naîtrait de la référence irreprésentable. Mais la pensée de Blanchot serait alors trop facilement saisissable; il ne faut pas amenuiser sa complexité, réduire ses paradoxes. Il est important ici de poser la distinction fondamentale entre la mort et la limite, la perte et l'absence, fracture où se joue toute la confrontation entre représentation et irreprésentable. L'absence demeure présente, il n'y a là aucune complaisance conceptuelle. Notre étude se charge en effet de mettre en valeur la disparition, absolu qui semble se désintéresser de son propre processus, celui de la perte. Elle serait, nous semble-til, la résolution de ce clivage de la perte et de l'absence qui en résulte. Le sujet du tableau. Pour montrer cela, il convient de révéler cet ajournement uniquement à l'intérieur de la mouvance de l'œuvre, c'est-à-dire de voir si le tableau se trouve, comme le Livre chez Blanchot, au-delà - en deçà? - ou au-dedans de la dialectique de l'image. Car si la tension subsiste, c'est entre l'opération qui entraîne la disparition et son aboutissement. La disparition: projet du maître, catastrophe permanente, ou ataraxie de l'œuvre qui n'en est jamais une? L'œuvre se meut, elle est ce mouvement même, celui de la rupture de sa propre forme, à perpétuité. Mais une rupture peut-elle être permanente? Ou plutôt, la cassure peut-elle échapper à la nécessité de faire œuvre?

La fêlure n'existe que par l'écart creusé par la parole, l'entre-deux que bordent deux rives: celles du langage. Le fameux bord sans bord dont discute Derrida dans Parages serait peut-être un espace intransitif à l'intérieur du langage, qu'il soit pictural ou littéraire. Le Neutre? Le questionnement se veut tel jusqu'à l'infini. Car signaler la perte, le silence, cela doit avoir lieu en présence de l'objet de cette disparition. Nous en avons convenu, l'écriture se dit intransitive chez Blanchot, ceci équivalant au refus de la représentation. Peut-on de même stipuler que la disparition obéit à quelque condition préalable, quelque dictat? Se peut-il qu'elle soit autoréferentielle? La question est épineuse dans la 
littérature blanchotienne: il faut concevoir le langage comme image, lieu de l'imaginaire ou en deçà de la parole, nous y reviendrons, puis comme image de rien, reflet pur d'elle-même. Qu'en est-il de la toile Carré blanc sur fond blanc? Car à n'en pas douter un tel titre soulève la question du miroir, de la réflexion spéculaire du ton sur ton. Comme une interrogation à l'infini du texte sur lui-même.

Abolir la narration, vœu cher à Malevitch comme à Blanchot. Bannir tout élément littéraire, toute littérarité: l'œuvre n'est plus à lire mais à voir. Mais s'acharner à accomplir la présentation, non plus la représentation de l'absence: c'est d'abord laisser voir quelque chose, et non pas rien. Position délicate concernant le nihilisme. Apparemment, nos artistes ne cherchent pas l'au-delà du langage, plutôt son en deçà, là où toute création est en instance, en puissance, en devenir. Là où l'écriture n'est jamais, mais ne fait que commencer. Revenons à L'Espace littéraire: le silence y est encore sous la parole.

Écrire ne consiste jamais à perfectionner le langage qui a cours, à le rendre plus pur. Écrire commence seulement quand écrire est l'approche de ce point où rien ne se révèle, où au sein de la dissimulation, parler n'est encore que l'ombre de la parole, langage qui n'est encore que son image, langage imaginaire et de l'imaginaire, celui que personne ne parle, murmure de l'incessant et de l'interminable auquel il faut imposer silence si l'on veut, enfin, se faire entendre. 4

Le discours de l'œuvre - picturale, littéraire - ne se charge pas de mettre en lumière. Au contraire de Freud qui citait un enfant de trois ans que le noir effrayait et qui demandait qu'on lui dise quelque chose car "...du moment que quelqu'un parle, il fait clair" ${ }^{5}$, il se trouve selon nous que l'approche de l'œuvre, qui est l'œuvre elle-même pour Blanchot, est rencontre de l'opacité, de l'obscurité du noyau qui décime le silence. Le noir est intarissable. Ainsi est-il absent de la toile de Malevitch de 1918. Le blanc de la surface semble avoir dissipé toute ténébreuse parole pour faire advenir le silence. Celui-ci s'engendre de l'évacuation de la mimésis, de la disparition figurée du quadrilatère. Mais à nouveau, la disparition n'est- elle plus un après-coup? Peut-elle faire fi de quelque antériorité? Le langage chez Blanchot, nous le répétons, se veut image donc simulacre: il est multiple. Il signe la perte de la référentialité, la mort $\mathrm{du}$ discours. Plus rien ne se déroule, et pourtant

[...] l'écriture ne naît pas du rapport heureux, d'immédiateté ou de maîtrise, avec les choses, mais [l'écrivain] rompt avec celui-ci, non pour accéder à une fusion plus profonde ou plus essentielle mais au contraire pour entrer dans le règne de l'Image, chaos, non $d u$ sens, où la ressemblance est ressemblance à rien. 6

Le simultané est une quête, d'où l'on est en droit de se demander s'il est jamais possible de (re)gagner ce silence, cette absence ex nihilo semblant être étrangement le fruit d'une certaine médiation. D'une façon que l'on sait admise, il n'est pas possible de figurer une perte, disparition, silence et effacement, sans en indiquer l'objet. La littérature blanchotienne se destitue en permanence d'un objet potentiel. De ce qui pourrait laisser trace. "Tout doit s'effacer, tout s'effacera. C'est en accord avec l'exigence infinie de l'effacement qu'écrire a lieu et a son lieu», dit-il 7 . Comment éviter la marque et qui plus est donner vie, corps à une ouvre, où, selon Blanchot encore: « $L a$ marque, c'est manquer au présent et faire que le présent manque» 8 ? C'est bien toujours rappeler le texte à sa quintessence: l'écriture. Le Livre est écriture, mais jamais au présent, temps de l'immédiateté. Elle vise l'éternel reflet, s'attache à l'impersonnalité, donc à l'intemporel. Dès lors, nous admettons que l'œuvre est mouvement à l'état pur. Au-dehors. Sans commencement ni fin, sans stagnation, il y a encore réflexion du mécanisme. Pour montrer l'infini, est-il meilleure image que celle de la chute, à l'intérieur d'un gouffre sans bords? Si l'écriture s'efface d'ellemême, ne peut-on dire que c'est au centre de l'abîme qu'elle survient, échappant à tout marquage puisque n'atteignant jamais le fond de cet abîme? Et le vide s'offre en imprescriptible, au sens où rien ne réussit à s'y inscrire.

Nous l'avons déjà évoqué, le langage pour Blanchot constitue un écart, une différance déplaçant indéfiniment le noyau de l'œuvre dans une zone 
obscure. Aucun point de chute, point mortel d'où auteur et œuvre ne réchappent, origine ou infini, et pourtant:

Ce point est l'exigence souveraine, ce dont on ne peut s'approcher que par la réalisation de l'œuvre, mais dont seule aussi l'approche fait l'ouvre. Qui ne se soucie que de brillantes réussites est pourtant à la recherche de ce point où rien ne peut réussir. Et écrit par seul souci de la vérité, est déjà entré dans la zone d'attirance de ce point d'où le vrai est exclu. Certains, par on ne sait quelle chance ou malchance, en subissent la pression sous une forme presque pure: ils se sont comme approchés par hasard de cet instant et, où qu'ils aillent, quoi qu'ils fassent, il les retient. Exigence impérieuse et vide, qui s'exerce en tout temps et les attire hors $d u$ temps. 9

Descente vers la profondeur du vide qui n'est en fait que surface: le lieu où Eurydice est précipitée est inatteignable, imprésentable, donc irreprésentable. Ce que le regard scrutateur ne peut fouiller demeure intangible, pour reprendre l'idée de Merleau-Ponty selon laquelle "Toute vision s'effectue dans l'univers tactile». Serait-ce possible de représenter une surface? N'y a-t-il pas ici défaillance du signe à l'endroit où ne subsiste que l'écran du simulacre, le plus parfaitement plan? Nous touchons finalement au problème de la limite. Le langage, la déchéance sont hors limite puisque étant eux-mêmes représentation, ceci du fait de la distance par où ils parlent et qui rétablit le champ de la médiation, en dépit des tentatives pour les en affranchir. Or, il se trouve que la limite est, par essence, irreprésentable. Est-ce le cas de la mort? «La mort, métaphore de la nomination, est une métaphore de ce mythe du langage même, écrit Henri Meschonnic ${ }^{10}$ avant d'ajouter: [...] D'une certaine façon et depuis toujours, nous savons que la mort est une métaphore pour nous aider à nous représenter grossièrement l'idée de limite, alors que la limite exclut toute représentation, toute "idée" de limite»" 11. La mort est toujours représentée car je ne meurt jamais. Elle est donc toujours autre. Toujours opérante et présente, mais jamais en dehors de celui qu'elle sectionne: le langage. Il s'agirait du différend, mouvement d'éloignement et de fraction de l'unité du langage. Cette unité adamique dont parle aussi
W. Benjamin, celle d'avant la chute, d'avant le péché originel. La puissance du fragmentaire est par ce principe toujours à l'œuvre. (Elle reste cependant subsidiaire face à la prédominance de la configuration de circularité de l'œuvre blanchotienne.) Ce pouvoir de déracinement, motif récurrent et obsédant qui conduit au nomadisme, est signe de l'effacement comme du désœuvrement. Il porte témoignage de cette dissémination originaire que la parole fait subir au langage. "[...] il y a une violence originaire de l'écriture parce que le langage est d'abord [...] écriture. L'usurpation" a toujours déjà commencé», cite Sarah Kofman*. Autant dire que rien ne précède ce déplacement. Comme si la représentation précédait, de manière intransitive. (Baudrillard parlerait de "précession des simulacres»). De fait, elle ouvre son domaine à la mort:

La mort se donne à penser sous la catégorie du vol. Elle n'est pas ce que nous croyons pouvoir anticiper comme le terme d'un processus ou d'une aventure que nous appelons -assurément- la vie. La mort est une forme articulée de notre rapport à l'autre. Je ne meurs que de l'autre: par lui, pour lui, en lui. Ma mort est représentée, qu'on fasse varier ce mot comme on voudra. Et si je meurs par représentation à la "minute de la mort extrême», ce dérobement représentatif n'en a pas moins travaillé toute la structure de mon existence, depuis l'origine. 12

À partir de ces notions de représentation, ce qui nous intéresse est d'étudier la démarche de Malevitch dans le Carré blanc sur fond blanc: a-t-il problématisé la question de la limite par rapport à celle de la mort de l'art? Comment a-t-il entrepris de tarir le langage pictural? Si ces questions se soulèvent dès les années 1910, c'est que l'abstraction cherche déjà à produire des œuvres par le concept de l'économie de signe, une certaine idéologie anti-verbale. L'enjeu artistique des avant-gardes, celui de l'art moderne en particulier, devient de déplacer le sujet de l'œuvre à son médium. Le modernisme s'affirme dans ce procédé que $\mathrm{R}$. Barthes nommait "étrange strabisme d'une opération en boucle». La peinture moderne s'acharne à discréditer sans exception les lois de la perspective et poursuit frénétiquement la recherche de l'aplatissement des formes et de la texture de la pâte à

* Lectures de Derrida, Paris, Galilée, 1984, p. 101. 
la surface de la toile. C'est la mort de Cézanne, survenant en 1906, qui forge le fer de lance à l'épopée de la planéité. Condamner la représentation oblige à aplanir les contours de l'objet en dépossédant la vertu du voir de ses attributs d'illusionnisme. L'art classique, depuis la Renaissance, donnait à croire que le visible manifesté sur le tableau était sans limite, c'est-à-dire qu'il semblait rendre compte de la réalité d'un paysage par l'ordonnancement de l'œil. Le cadre délimitait de manière extrinsèque la leçon de la vision. Au XXe siècle, l'art se détourne de l'œuvre de la vision pour ne s'intéresser qu'au geste de voir. Même aventure pour la littérature, où l'instrumentalisation du langage est réprouvée. $\mathrm{Ne}$ subsiste qu'une langue mise à nu que l'auteur découvre, ou tente de redécouvrir. Le geste pictural de Malevitch s'informe donc de cet esprit de dévoilement, de cette mission d'éclipse des sujets et des objets. De ce retour à une certaine quintessence de l'œuvre en tant qu'infinie question sur elle-même. Quelle source d'inspiration donc, si ce n'est la picturalité elle-même, le pictural plutôt en tant que tel? Car rappelons que pour Blanchot, ce qu'il nomme «inspiration» est d'abord «ce point pur où elle manque». L'art de Malevitch se constitue de cette approche du lieu de l'œuvre en exil; pour tout dire, il est désir d'y disparaitre, de plonger dans la surface. Le blanc de la toile part en quête de planéité car celle-ci manque toujours un peu. Comme l'art de Mondrian, qui avait l'habitude de se plaindre que le blanc ne savait pas être assez plat. Le Suprématisme est la recherche de l'absolu de la couleur et de la forme en tant que cet absolu est la limite de l'art: un art dont l'éloquence se tarit finalement dans le blanc sur blanc, qui déconstruit la représentation de la limite pour la faire exister dans l'absence de démarcation. Car si la limite exclut toute représentation, comment dire que c'est d'elle qu'il s'agit dans le tableau de Malevitch? Selon nous, la mort de l'art telle qu'elle se problématise là n'est pas de l'ordre du mutisme: au contraire, elle est de l'ordre du cri. C'est un silence, espace blanc infini et vibrant de matière «lumière» qui hurle à la surface de la toile. C'est la lumière qui se veut effacement de l'écriture picturale. Elle qui n'est plus révélatrice de rien, tout comme le langage chez Blanchot, en qui la mort fait son ouvre et rompt la communication. Non plus éclat du dévoilement, mais éclairement de l'absence, de l'indicible. La fin de l'art est-elle impossible à représenter? Il nous semble que cette question n'équivaut nullement à celle de savoir si la fin de l'art peut déjà être proclamée. Comme l'écrivait Octavio Paz à propos de la modernité, «[...] nous vivons la fin de l'idée d'art moderne", sous-entendant que l'art en soi n'en était pas encore à son dernier souffle.

"L'essence du langage consisterait à passer du langage à l'indicible qui se dit, à rendre visible par l'œuvre l'obscurité de l'élémental», écrit E. Lévinas ${ }^{13}$. En effet, la peinture pour Malevitch n'est plus là pour dire, mais pour montrer, montrer qu' «il y a quelque chose qu'on ne peut pas voir», comme dit Lyotard. D'où une possible monstruosité reprochée à l'art abstrait, un art qui donne la vision, le voir indifférencié d'un réel sans contours, ramassé sur lui, plat et ainsi muet. La carence de perspective se noue à l'absence de fond de l'abîme: "L'intériorité est à jamais sans fond et se confond dans l'infini, à jamais irréalisable», écrit le peintre*. Cela justifie le ton sur ton: l'absence de distinction, de coupure, l'amorphe, qui est aussi de l'informe. Or il se trouve que le tableau de Malevitch n'est pas exempt de formalisme; de fait, le formalisme est ce qui équivaut au modernisme pour le critique d'art Clement Greenberg. La forme ici est exaltée: celle du carré, absolu, indéformable. Elle profile une tension qui rappelle combien l'énergétique est à la base de la peinture, même lorsque celle-ci, élevée à l'abstraction, veut l'épuisement des formes. "Ce sont les "forces" qui créent la forme, et ce sont elles qui importent, plus que la forme elle-même, le chemin plus que la fin, la genèse et le devenir plus que le résultat", explique A. Gutmann ${ }^{14}$. Il s'agit de voir si la figuration s'épuise dans cette lutte que signifie la pratique de l'abstraction: ab-straire, ou étymologiquement «se retirer». S'éclipser du «monde comme volonté de représentation", ou comment être un peintre pur, c'est-à-dire se cantonner à polir la surface de la peinture dans la présentation terminale d'elle-

* Cité dans Cahier Malevitch, L’Âge d'homme, 1983, p. 177. 
même. Faire voir sa limite, là où le blanc le plus pur, la lumière la plus transperçante ne peut plus faire voir la transparence: car transparence de quoi, si ce n'est de rien? L'opacité à présent se fait entendre: échec du silence qui en voulant s'élaborer dans la transparence ne peut taire son éloquence naturelle. Le silence, tout comme l'abstraction, ne peut être intégral, s'il suppose une transitivité: «faire taire un bruit», «faire abstraction de quelque chose", de telles expressions ruinent tout mutisme, vide préalable, mais font au contraire référence à la qualité intrinsèque de l'art: son mouvement.

À la veille de la Révolution de 1918, le peintre russe se livre à une recherche sur la structure fonctionnelle de l'image. Désirant parvenir à un degré d'abstraction totale, il entreprend de passer au stade de la non-objectivité, qui désigne un Monde sans Objet - ce sont ses propres termes et le titre de son manifeste de 1922 - pour un monde sans passé ni futur. "Et j'ai vu que l'objet et son image n'étaient qu'un reflet spéculaire de mes sensations, et j'ai percé le mensonge de ce monde de volonté et de représentation", écrit-il". L'effort souverain sera alors de porter l'objet à la limite de sa représentation, au point suprême de ce vide conçu comme une réalité cosmique. Lieu où l'intériorité est vaincue. Il s'agit peut-être de cet état de «ruissellement $d u$ dehors» comme l'évoque Michel Foucault dans son texte sur Blanchot, L'Écriture $d u$ dehors. Le lieu aussi de la poésie par excellence, par où s'effectue toute expérience abyssale de la création face à l'absence d'inspiration. Blanchot en toute éminence s'apparente à celle du poète:

Mallarmé, qu'a tourmenté l'état de sécheresse et qui s'y est enfermé par une décision héroïque, a aussi reconnu que cette privation n'exprimait pas une simple défaillance personnelle, ne signifiait pas la privation de l'œuvre, mais annonçait la rencontre de l'œuure, l'intimité menaçante de cette rencontre. 15

Le peintre aussi doit se trouver aux abords de l'œuvre. Ou bien s'en éloigner. Le Suprématisme correspond à cet au-delà ou en deçà du point central de l'œuvre, de son origine inassignable, qu'il fuit

\footnotetext{
* Malevitch, Écrits I. Traités de 1915-1922, L'Âge d'homme, 1974, p. 191.
}

grâce à cet élan vers le "zéro de la forme», le silence en peinture. Malevitch conçoit peut-être déjà que le point mortel de l'œuvre est celui où la vision s'échoue, rendant manifeste la distance infranchissable, l'écart qui irréalise l'immédiateté, la proximité. Cette séparation dans laquelle pour Blanchot «tout parle», où l'écriture a rapport à l'incessant, à la dissimulation et la non-révélation. C'est encore l'Origine. Le dessein de l'artiste est tout autre: "dévoiler le rien", montrer la disparition des objets dans l'expérience d'une vision où s'affiche parfaitement le pouvoir de ce geste. Car voir, c'est perdre. Comme si le coup d'œil était responsable de ce qui affleure à la surface. Là est le défi. Faire voir le voir. Le regard signait déjà la perte dans le périple d'Orphée. Il reste cette instance maudite à même le tableau. Peindre un regard: le figer ou lui rendre sa mobilité? Sans objet, point de mobile. La peinture de Malevitch a ceci de brillant qu'elle s'accole au mouvement. "L'art est la capacité de créer une construction qui ne découle pas des relations entre les formes et la couleur [...] mais qui est bâtie sur le poids, la vitesse et la direction $d u$ mouvement", explique-t-i ${ }^{16}$. Le problème de la quatrième dimension n'est qu'accessoire et dérivé. C'est la cinquième dimension ou économie qui intéresse Malevitch. Il faut montrer le Carré en train de disparaître. Gommé par la suprématie de l'espace. La platitude du carré est bien un rappel de cette caractéristique d'un espace sans fond, celui de l'infini. Le blanc en peinture, non-couleur absente de la nature, est l'élément de lumière, la visibilité pure. Il est aussi le sémaphore du Suprématisme, là où toute teinte est vaincue en raison de l'absence de formes. Cette matière est alors silence. Luminosité aveuglante du tissu de l'œuvre chez Blanchot, elle est la rencontre de ce point de dispersion, celui de la fureur iconoclaste de Malevitch où l'imitation est résiliée. Une sorte de tache aveugle paradoxalement. L'art moderne affiche sa volonté de silence. Il faut montrer, comme le dit Lyotard, qu'il y a de l'imprésentable, quelque chose que l'on ne peut pas voir. Mais cet imprésentable n'est pas synonyme d'irreprésentable: au contraire, l'échec de l'abstraction suprême, de l'art moderniste, réside nous semble-t-il dans cette 
annexion à la représentation. Pour ce faire, le peintre russe ne souhaite pas peindre l'invisible. Cela, il en a conscience, est voué à l'échec. Comment pourrait-on peindre une surface, un espace? Ce qu'il faut, c'est que l'espace lui-même vienne à se peindre sur la toile. L'autofiguration dont nous avons ébauché l'idée plus haut est l'outil de l'abstraction. (Elle est aussi la condition de l'objectivité de l'œuvre.) Celle-ci ordonne de rendre visible la disparition du visible. Le Carré blanc sur fond blanc est le signe de l'effacement. Il est donc aussi la trace de son effacement supposé progressif, le signe d'un désir de pureté qui ôte l'élément tactile de la toile et lui rend une matérialité optique. L'éclipse de l'objet ne fait pas sens, ne dit pas la mise en forme de l'espace; c'est l'espace qui préside et préfigure l'évacuation du carré pour exprimer l'autorité du support. Le plan devenu non instrumental marque alors le raccourci conceptuel de l'art moderne. Le réductionnisme est en marche, tant chez Malevitch que chez Blanchot: il s'efforce d'aboutir à la complexité de l'œuvre, c'est-à-dire au lieu de son silence.

Il ne faut pas anticiper l'advenue du silence. L'art moderne, avant de vouloir ne rien dire, vise à dire le rien. La sphère de négativité dans laquelle l'œuvre évolue n'est pas refus des possibilités de l'art, mais affirmation de son infini. Un infini des possibles que le blanc offre, signe d'une peinture en devenir. L'artiste exclu de son ouvre, la peinture exclue de sa toile, que reste-t-il sinon une potentialité pure, un devenir que nul regard ne peut appréhender? L'absence d'objet ne décourage pas le regard; au contraire, il faut écouter Blanchot qui allègue que:

Le moment exceptionnel où la possibilité devient pouvoir, où, loi et forme vide qui n'est riche que de l'indéterminé, l'esprit devient la certitude d'une forme réalisée, devient ce corps et cette belle forme qui est un beau corps. L'œurre est l'esprit, et l'esprit est le passage, en l'œuvre, de la suprême indétermination à l'extrême indéterminé. 17

Toute la naissance de l'art moderne semble concourir à cette esthétique de la simplification de la forme par la ligne, telle la recherche par exemple chez
Klee du petit «point gris», celui du silence où l'œuvre se dissimule, mais s'offre tous les potentiels.

L'évidement ou l'apologétique de la lumière signifie la vibration à l'infini de l'incréé qui coïncide avec son dépassement.

[...] pour Cézanne le blanc est la capacité lumineuse de toute

couleur: c'est le blanc, non comme couleur mais comme vide. Ce vide énergétise les tensions jusqu'à leur limite tectonique qui est le moment de décision de l'œurre, son événement. [...] c'est le vide qui agit et articule la tectonique des mouvements (voir les "Sainte-Victoire»). 18

De même pour Derrida, il s'agit de la manifestation du nihil créateur, de cet «indéterminé déterminant». Si Malevitch semble cautériser le néant et l'infini, c'est pour mieux rendre manifeste la pulsation énergétique de la surface. "La présence permanente du néant garantit cette intensité maximale de l'existence de l'être pictural: vie et mort se côtoient dans une extraordinaire intensité", rapporte-t-il ${ }^{19}$. Cette démarche absolutiste correspond à une conception dynamique de la matière (couleur, plan et aussi écriture, chair des mots), qui s'élabore dans une constante métamorphose. Le mouvement d'effacement, de retrait du Carré qui n'en finit pas de disparaitre, est ce flux et reflux, dialectique de l'image, dualité de l'instance créatrice: gestation et destruction. À l'instar de la beauté dans l'œuvre de Breton: «explosante-fixe», celle-là peut-être même de la dialectique de l'image, en qui le mouvement de l'écart de l'origine, la différance, constitue la vérité du tableau, la vérité du texte.

Un infiniment plat est aussi manière de conjurer le vide, de déceler le tout. Comme le rejet du vide au creux des mots de la littérature blanchotienne. Des mots pour dire le silence, ou bien un silence à l'intérieur des mots? Une peinture du silence, ou bien un silence en peinture? Les questions, affirmons-le d'emblée, ne sont pas simplistes. Il y va de toute la question du sens. La problématique, semble-t-il, ne s'est point résorbée. C'est qu'il s'agit toujours de dire, non de raconter. Dire un silence, pour ne pas raconter un tarissement. Une épreuve simultanée. L'aveu de l'impossible se fait déjà entendre: 
Comment dire ce qui ne porte pas de limite, pas de frontière, entre le pensé et l'impensable, entre le dedans et son dehors, entre l'envers et l'endroit? Le langage ne peut pas naître lorsqu'il n'y a pas de trace de limite. Ce qui ne se représente pas ne se "représente» pas. Les mots et les paroles qui viennent au secours des images représentent, le silence reste en deçà de la «représentation", en deçà de la répétition. Comment répéterait-on ce qui ne se nomme pas? Le silence ne se répète pas, il est. Seuls les mots pour le dire le répètent, le rappellent. (L. Laufer) ${ }^{20}$

Comment éviter de dire le silence? Le montrer est déjà un geste. L'on convient que le nihil ne peut être créé. Il doit présider à toute présentation, voire représentation. En effet, il ne saurait être lui-même le sujet de l'œuvre, celui qui vient après. Le silence n'est point le donné du visible. Toute donation - l'écriture et la peinture en sont - signe la perte de référent. Si ce référent se trouvait être la non-communication, il faut alors que celle-ci s'auto-manifeste: par le style, modalité de l'expérience littéraire et picturale, le ton qui se dit lui-même, comme pour Michel Foucault, chez qui la folie, nous dit Derrida, se raconte ellemême:

On pourrait peut-être dire que la solution de cette difficulté est plus pratique que formulée. Par nécessité. Je veux dire que le silence de la folie n'est pas dit, ne peut pas être dit dans le logos de ce livre mais rendu présent indirectement, métaphoriquement si je puis dire, dans le pathos - je prends ce mot dans son meilleur sens - de ce livre. Nouvel et radical éloge de la folie dont l'intention ne peut s'avouer que parce que l'éloge d'un silence est toujours dans le logos, dans un langage qui objective; "dire-du-bien-de» la folie, ce serait encore l'annexer surtout lorsque ce "dire-du-bien-de» est aussi, dans le cas présent, la sagesse et le bonheur d'un «bien-dire». 21

La donation du visible est le sensible: ce dernier est déjà le sentiment de l'absence, la sensation que l'origine de la picturalité, celle qui prévaut au langage de la peinture, ne peut se donner à voir. C'est cela le propre de l'abstraction: donner le retrait, l'aperçu de la trace du langage qui partant est vacant. La fuite du carré de Malévitch, son départ de la toile est toujours la marque d'un insignifiable. Un sens du tableau différé par le système d'attente qu'érige le langage plastique. Car enfin, «un tableau ne veut rien dire» répète Sarah Koffman ${ }^{22}$. L'issue du questionnement se trouve sans doute ici: le silence doit rester un insu, ainsi qu'un «invu». Qu'il se dévoile et déjà sa signifiance le constitue en trop-plein. Le silence est en défaut: toujours signifiant, il est contraint de s'autodétruire. Il est l'être du manque, parfaitement innommable. Impasse désespérante du créateur qui ne veut rien inventer, mais tout laisser advenir. Peindre un silence, c'est rendre une toile illisible. Abolir son sens: veiller à ce qu'il n'y ait rien à décrypter. Le tableau n'a plus ni envers ni endroit, c'est-à-dire deux côtés, dont l'un serait celui du maître, l'autre celui du voyeur. Une seule et même face subsiste: en témoigne la transparence voulue, désirée, d'un carré qui laisse voir à son travers le plan qui le supporte, ou l'engloutit. Surface insondable d'un fond sans fond: métaphore encore d'un silence mis en échec par l'impossible de cette spécularité. Effectivement, l'opacité est toujours à tenter de défaire; de là, elle produit du discours. Le blanc sur blanc en appelle bien trop au mystère pour parvenir à empêcher toute tentative allusive, métaphorique. Ce blanc est donc un voile: on ne sait si l'intérieur du carré est la surface du carré lui-même, ou l'espace qui en déborde les côtés. Du reste, le blanc peut bien être à la fois transparent ou opaque. L'important est de dire que, de ces deux qualités, le silence ne perce pas. La vision est déchirée par le sensible: il lui fait la violence de dire, et provoque le déchirement. La parole est alors blessure de la vision. La déhiscence d'où elle naît est déjà audelà du silence. C'est cela que le peintre comme l'écrivain veulent affronter: l'énigme de l'œuvre dont l'objet excède ses auteurs, comme ses exégètes. La peinture moderne déjoue le commentaire. Mais son mystère tuant le silence, malheureusement, elle forme néanmoins l'œuvre de l'indicible. L'exemple de Cézanne est à ce titre éloquent:

Toutes les montagnes Sainte-Victoire peintes par Cézanne sont

là, comme un corps libidinal critique, absolument muettes, vraiment impénétrables parce qu'elles ne cachent rien, c'est-à-dire parce qu'elles n'ont pas leur principe d'organisation en dehors 
d'elles-mêmes (dans un modèle à imiter, dans un système de règles à respecter), impénétrables parce que sans profondeur, sans signifiance, sans dessous. 23

En revenant au concept de planéité, nous sommes amené à expliquer comment la révocation de l'héritage de la Renaissance est vecteur de silence. L'abstraction en effet ne se débarrasse pas toujours de la ligne. Nous l'avons vu avec Malevitch, la présence d'une masse, plutôt qu'une forme, au centre de l'œuvre, ne se justifie pas de l'existence d'un contour, d'une limite. Le carré donne l'illusion de son déploiement énergétique par la matière picturale proprement dite. La densité de cette matière vibrante utilise en quelque sorte la possibilité de son apparence autonome pour poser la question fondamentale de l'existence de la couleur: par la lumière, ou bien par la forme? Question irrésolue selon nous, aporétique, que celle de la représentation, si ce n'est en tenant compte des lois de la gravité et du mouvement. C'est par la sensation du poids et de la vitesse, nous l'avons dit, que l'artiste russe s'efforce de montrer un carré, non pas d'en imiter l'apparence. L'apparition de la forme abolit la mimésis par son mouvement même. C'est aussi pour Malevitch une manière de résoudre grâce à l'espace le problème du figurable. Par sa préexistence, il permet la présentation avant même la représentation. Le retournement des lois de la perspective, celles qui prônaient la dépendance du fond par rapport à la forme, rend à la lumière son statut inaugural. La couleur n'a plus qu'un aspect symbolique, significatif. Le blanc, le noir, comme noncouleur, associent paradoxalement lumière à épaisseur. Or voilà où le bât blesse: en donnant du poids à la forme en instance de disparition, pour exprimer ce phénomène-ci, il annule la transparence désirée de l'absence de support, et recrée une épaisseur à tarir. C'est cela que semble dire sans le vouloir la toile Carré blanc sur fond blanc: qu'il n'y a point d'absence de voile. Que tout dévoilement du rien n'établit pas le vide, le silence, mais l'absence de vacuité.

\section{N O TES}

1. Remarque de L. Ferry citant Malevitch, dans Homo aestheticus, Paris, Grasset, 1990, p. 274.

2. M. Blanchot, L'Espace littéraire, Paris, Gallimard, 1947, p. 231.

3. J. Pfeiffer, "Maurice Blanchot", Critique, no 229, juin 1966, p. 572.

4. L'Espace littéraire, p. 48.

5. Citation d'une note des Trois Essais sur la théorie de la sexualité, extraite de l'article de L. Laufer, "J'ai fait silence», dans Le Silence, collectif de la série "Autrement», coll. "Mutations", n 185, avril 1999 , p. 51

6. F. Collin, dans Critique, no 229, déjà citée, p. 566.

7. M. Blanchot, Le Pas-au-delà, Paris, Gallimard, 1973, p. 76.

8. Ibid., p. 77.

9. L'Espace littéraire, p. 56-57.

10. H. Meschonnic, "Maurice Blanchot ou l'écriture hors langage", dans Pour la poétique, vol. 5, Paris, Gallimard, 1970.

11. Le Pas-au-delà, p. 75

12. J. Derrida, L'Écriture et la Différence, Paris, Seuil, 1967, p. 268-269.

13. E. Lévinas, Sur Maurice Blanchot, Paris, Fata Morgana, 1975, p. 18

14. A. Gutmann, "Entendre la peinture», dans Le Silence, déjà cité à la note 5 , p. 135.

15. L'Espace littéraire, p. 233.

16. K. Malevitch, Écrits, Paris, Éd. Gérard Lebovici, 1986, p. 92.

17. M. Blanchot, "À toute extrémité", NRF, 1955, p. 288.

18. P. Fédida, L'Absence, Paris, Gallimard, 1978.

19. K. Malevitch, Écrits, p. 101.

20. L. Laufer, dans Le Silence, p. 30.

21. J. Derrida, L'Écriture et la Différence, p. 60.

22. S. Kofman, Mélancolie de l'art, Paris, Galilée, 1985, p. 22.

23. J.-F. Lyotard, Des dispositifs pulsionnels, "Freud selon Cézanne», Paris, Galilée, 1994, p. 86. Cité dans l'article de R. Steinmetz, «J.-F. Lyotard: le silence en peinture ", L'Image: Deleuze, Foucault, Lyotard, Paris, J. Vrin, 1997, p. 28. 